\title{
Surface Criticality and Multifractality at Localization Transitions
}

\author{
A. R. Subramaniam, ${ }^{1}$ I. A. Gruzberg, ${ }^{1}$ A. W. W. Ludwig, ${ }^{2}$ F. Evers, ${ }^{3}$ A. Mildenberger, ${ }^{4}$ and A. D. Mirlin ${ }^{3,5, *}$ \\ ${ }^{1}$ James Franck Institute, University of Chicago, 5640 South Ellis Avenue, Chicago, Illinois 60637, USA \\ ${ }^{2}$ Physics Department, University of California, Santa Barbara, California 93106, USA \\ ${ }^{3}$ Institut für Nanotechnologie, Forschungszentrum Karlsruhe, 76021 Karlsruhe, Germany \\ ${ }^{4}$ Fakultät für Physik, Universität Karlsruhe, 76128 Karlsruhe, Germany \\ ${ }^{5}$ Institut für Theorie der kondensierten Materie, Universität Karlsruhe, 76128 Karlsruhe, Germany
}

(Received 2 December 2005; published 27 March 2006)

\begin{abstract}
We develop the concept of surface multifractality for localization-delocalization (LD) transitions in disordered electronic systems. We point out that the critical behavior of various observables related to wave functions near a boundary at a LD transition is different from that in the bulk. We illustrate this point with a calculation of boundary critical and multifractal behavior at the 2D spin quantum Hall transition and in a $2 \mathrm{D}$ metal at scales below the localization length.
\end{abstract}

DOI: 10.1103/PhysRevLett.96.126802

Localization-delocalization (LD) transitions in disordered electronic systems represent a remarkable class of quantum phase transitions. One of their striking features is that moments of wave functions near a LD transition are characterized by a set of independent critical exponents [1]. Criticality of the wave functions manifests itself in their multifractality (MF) [2,3], which has been studied analytically and numerically for a variety of systems: the Anderson transition in $d=2+\epsilon, 3$, and 4 dimensions [1,4], as well as weak MF [5], random Dirac fermions [6], integer quantum Hall (IQH) [7], spin quantum Hall (SQH) [8] transitions in two dimensions, and power-law random banded matrices [9].

It is known that at conventional phase transitions, different critical behavior occurs at a boundary, as compared to the bulk of the sample [10]. This is especially well understood in two dimensions where methods of conformal field theory (CFT) are available [11].

In this Letter we propose to extend the concept of surface criticality to LD transitions. Such an extension has several potentially important applications. First, LD transitions are studied experimentally mainly through transport measurements, which are performed by attaching leads to the boundary of a finite sample. With such a setup it is feasible to measure surface critical behavior directly. Second, we will show that even if the MF of wave functions in the whole sample is studied, the surface effects are fundamentally important. Further, LD transitions in two dimensions are expected to be described by certain CFTs. Such a description remains elusive for the primary example of the IQH transition, though some proposals have been put forward $[12,13]$. Studying the MF of wave functions near a surface is expected to help identify the CFT for the IQH transition and similar systems.

Let us start with a brief review of bulk MF in the context of $\mathrm{LD}$ transitions. The MF of wave functions $\psi(\mathbf{r})$ at a LD transition is characterized by the scaling of moments of $|\psi(\mathbf{r})|^{2}$ with system size $L$ :
PACS numbers: 73.20.Fz, 72.15.Rn, 73.43.-f

$$
L^{d}\left\langle|\psi(\mathbf{r})|^{2 q}\right\rangle \sim L^{-\tau_{q}}, \quad \tau_{q} \equiv d(q-1)+\Delta_{q},
$$

where $\langle\cdots\rangle$ denotes the disorder average. Note than one often introduces fractal dimensions $D_{q}$ via $\tau_{q}=D_{q}(q-1)$. In a metal $D_{q}=d$, while at a critical point $D_{q}$ is a nontrivial function of $q$, implying the MF of wave functions. Nonvanishing anomalous dimensions $\Delta_{q} \equiv(q-1)\left(D_{q}-\right.$ d) distinguish a critical point from a metallic phase and determine the scaling of wave function correlations. Among them, $\Delta_{2}<0$ plays the most prominent role, governing the spatial correlations of the intensity $|\psi|^{2}$ : $L^{2 d}\left\langle\left|\psi^{2}(\mathbf{r}) \psi^{2}\left(\mathbf{r}^{\prime}\right)\right|\right\rangle \sim\left(\left|\mathbf{r}-\mathbf{r}^{\prime}\right| / L\right)^{\Delta_{2}}$. This equation, which in technical terms results from an operator product expansion of the field theory [14], can be obtained from (1) by using the fact that the wave function amplitudes become essentially uncorrelated at $\left|\mathbf{r}-\mathbf{r}^{\prime}\right| \sim L$. Scaling behavior of higher order spatial correlations, \langle|$\psi^{2 q_{1}}\left(\mathbf{r}_{1}\right) \times$ $\psi^{2 q_{2}}\left(\mathbf{r}_{2}\right) \cdots \psi^{2 q_{n}}\left(\mathbf{r}_{n}\right)|\rangle$, can be found in a similar way. Above, the points $\mathbf{r}_{i}$ were assumed to lie in the bulk of a critical system. In this case we denote the multifractal exponents by $\tau_{q}^{\mathrm{b}}, \Delta_{q}^{\mathrm{b}}$, etc.

In finite electronic systems, wave functions vanish on the boundary. Therefore, to assign a meaning to the MF on the boundary, we have to interpret surface correlation functions in the sense of the theory of critical phenomena $[10,11]$. Specifically, by "points on the boundary" we mean points that lie close to it, as compared to other distances, e.g., a few lattice spacings away.

With this caveat in mind, we now generalize the notion of MF to systems with boundaries. First, we note that in general even the average value of $|\psi(\mathbf{r})|^{2}$ for $\mathbf{r}$ at the boundary may be nontrivial, $\left\langle|\psi(\mathbf{r})|^{2}\right\rangle \propto L^{-d-\mu}$. In particular, $\mu$ can be nonzero in systems with unconventional (chiral or Bogoliubov-de Gennes) symmetry [15], which are attracting particular interest in connection with physics of disordered superconductors. For the moments of the intensity, we get in analogy with Eq. (1)

$$
L^{d-1}\left\langle|\psi(\mathbf{r})|^{2 q}\right\rangle \sim L^{-\tau_{q}^{s}},
$$




$$
\tau_{q}^{\mathrm{s}}=d(q-1)+q \mu+1+\Delta_{q}^{\mathrm{s}}
$$

with a new set of surface multifractal exponents $\tau_{q}^{\mathrm{s}}, \Delta_{q}^{\mathrm{s}}$, which are in general independent of their bulk counterparts [16]. The normalization factor $L^{d-1}$ is chosen such that Eq. (2) yields the contribution of the surface to the inverse participation ratios $\left\langle P_{q}\right\rangle=\left\langle\int d^{d} \mathbf{r}|\psi(\mathbf{r})|^{2 q}\right\rangle$ conventionally studied in the framework of the MF analysis. The exponents $\Delta_{q}^{\mathrm{s}}$ as defined in Eq. (3) vanish in a metal and govern statistical fluctuations of wave functions at the boundary, $\left\langle|\psi(\mathbf{r})|^{2 q}\right\rangle /\left\langle|\psi(\mathbf{r})|^{2}\right\rangle^{q} \sim L^{-\Delta_{q}^{\mathrm{s}}}$, as well as their spatial correlations, e.g., $L^{2(d+\mu)}\left\langle\left|\psi^{2}(\mathbf{r}) \psi^{2}\left(\mathbf{r}^{\prime}\right)\right|\right\rangle \sim\left(\left|\mathbf{r}-\mathbf{r}^{\prime}\right| / L\right)^{\Delta_{2}^{s}}$.

In fact, these notions can be further generalized. First, for multipoint correlation functions, some points may be in the bulk while the rest are on the surface. Then the scaling behavior will be described by mixed bulk-surface exponents. Second, surface and mixed exponents will sensitively depend on the global geometry of the boundary. For example, if the point $\mathbf{r}$ in Eq. (2) lies near the edge of a wedge with the opening angle $\theta$, multifractal exponents will continuously depend on $\theta$. We relegate the analysis of these generalizations to a future publication [17] and concentrate here on the fundamental surface exponents $\tau_{q}^{\mathrm{s}}, \Delta_{q}^{\mathrm{s}}$.

We now illustrate these points in the case of the 2D SQH plateau transition, which belongs to symmetry class C (in the classification of Ref. [15]), relevant for the description of quasiparticle transport in singlet superconductors with broken time-reversal symmetry [18]. A remarkable feature of the SQH effect is that a number of basic observables can be calculated exactly, as was discovered in Refs. [19,20], via a mapping of the corresponding network model [21] (similar to the IQH network of Ref. [22]) to the problem of classical percolation. This mapping was extended in Ref. [8] to extract analytical values of the bulk exponents $\Delta_{2}^{\mathrm{b}}=-1 / 4, \Delta_{3}^{\mathrm{b}}=-3 / 4$.

In what follows, we generalize this mapping to the $\mathrm{SQH}$ network with a boundary and use it to extract new surface critical and multifractal exponents. The SQH network consists of directed links $r$ that carry doublets of complex fluxes $\psi_{\alpha}(r)$ representing propagation of spin $1 / 2$ particles. Effects of disorder are introduced through random SU(2) scattering matrices on the links. At each node the scattering from two ingoing to two outgoing links is described by the (spin-independent) matrix $S$, with $S_{11}=$ $S_{22}=\left(1-t^{2}\right)^{1 / 2}, S_{12}=-S_{21}=t$. The value $t=1 / \sqrt{2}$ corresponds to the critical point of the $\mathrm{SQH}$ transition.

In order to study the effect of boundaries, we impose reflecting boundary conditions along one direction. This does not affect the symmetry of the system, which enables us to retain the mapping to percolation. In technical terms, within the approach of Ref. [19], supersymmetry (SUSY) is preserved at the boundary nodes of the network model. We have also checked [17] that the SUSY method can be extended to the two- and three-point functions needed to extract $\tau_{q}^{\mathrm{s}}$ with $q=2,3$, and yields results identical with the path integral approach developed in Refs. $[8,20]$.
We calculate first the average local density of states (LDOS) at a point $\mathbf{r}_{1}$ on the boundary that will allow us to find the average of the intensity $\left|\psi_{\alpha}\left(\mathbf{r}_{1}\right)\right|^{2}$. The LDOS can be expressed in terms of one-point Green's functions and becomes, when mapped to percolation,

$$
\left\langle\rho\left(\mathbf{r}_{1}, \boldsymbol{\epsilon}\right)\right\rangle=(1 / 2 \pi)\left[1-\sum_{N} P\left(\mathbf{r}_{1} ; N\right) \cos 2 N \boldsymbol{\epsilon}\right],
$$

where $\epsilon$ is the energy and $P\left(\mathbf{r}_{1} ; N\right)$ is the probability of a $N$-link hull passing through $\mathbf{r}_{1}$, in analogy with [20]. The corresponding surface critical exponent is $x_{1}^{\mathrm{s}}=1 / 3$ [23], which should be contrasted to its bulk value $x_{1}^{\mathrm{b}}=1 / 4$. The latter value implies that the percolation hull has fractal dimension $2-x_{1}^{\mathrm{b}}=7 / 4$, so that $P(\mathbf{r}, N) \sim N^{-8 / 7}$ for $\mathbf{r}$ in the bulk. This yields, according to Eq. (4), the density of states scaling $\rho(\epsilon) \propto \epsilon^{x_{1}^{b} /\left(2-x_{1}^{b}\right)}=\epsilon^{1 / 7}[19,20]$. Note that $2-x_{1}^{\mathrm{b}}=7 / 4$ is the dynamic exponent governing the scaling of energy with the system size $L$ at $\mathrm{SQH}$ criticality, so that the level spacing at $\epsilon=0$ (and thus the characteristic energy of critical states) is $\delta \sim L^{-7 / 4}$.

In our case, when the point $\mathbf{r}_{1}$ is located at the surface, we find $P\left(\mathbf{r}_{1}, N\right) \sim N^{-1-x_{1}^{s} /\left(2-x_{1}^{b}\right)}=N^{-25 / 21}$ and the LDOS scaling $\rho\left(\mathbf{r}_{1}, \boldsymbol{\epsilon}\right) \propto \epsilon^{x_{1}^{s} /\left(2-x_{1}^{b}\right)}=\epsilon^{4 / 21}$. For the wave function at the boundary, this implies

$$
L^{2}\left\langle\left|\psi_{\alpha}\left(\mathbf{r}_{1}\right)\right|^{2}\right\rangle \sim L^{x_{1}^{b}-x_{1}^{s}}=L^{-1 / 12} .
$$

Therefore, the average intensity of a critical wave function is suppressed at the boundary with the exponent $\mu=1 / 12$. A similar calculation for the conductance between two point contacts $\mathbf{r}_{1}$ and $\mathbf{r}_{2}$ located at the boundary [24] yields scaling with exponent $2 x_{1}^{\mathrm{s}}=2 / 3$,

$$
g_{\mathrm{pt}}^{s}\left(\mathbf{r}_{1}, \mathbf{r}_{2}\right) \propto\left|\mathbf{r}_{1}-\mathbf{r}_{2}\right|^{-2 / 3} .
$$

We turn now to the multifractal exponents. To calculate $\Delta_{2}^{\mathrm{s}}$, we consider the correlation function [8]:

$$
\begin{aligned}
(2 \pi)^{-2} \tilde{\mathcal{D}}\left(\mathbf{r}_{1}, \mathbf{r}_{2}, \epsilon_{1}, \epsilon_{2}\right)= & \left\langle\sum_{i j \alpha \beta}\left|\psi_{i \alpha}\left(\mathbf{r}_{1}\right)\right|^{2}\left|\psi_{j \beta}\left(\mathbf{r}_{2}\right)\right|^{2}\right. \\
& \left.\times \delta\left(\epsilon_{1}-\epsilon_{i}\right) \delta\left(\epsilon_{2}-\epsilon_{j}\right)\right\rangle .
\end{aligned}
$$

To study critical states, we take $\epsilon_{1,2}=0$ and broaden delta functions by $\delta$. Using the mapping to percolation, we relate (7) to percolation probabilities,

$$
\begin{aligned}
\tilde{\mathcal{D}}\left(\mathbf{r}_{1}, \mathbf{r}_{2}, z\right)= & 4 \sum_{N}\left(1-z^{2 N}\right) P\left(\mathbf{r}_{1}, \mathbf{r}_{2} ; N\right)+4 \sum_{N, N^{\prime}}\left(1-z^{2 N}\right) \\
& \times\left(1-z^{2 N^{\prime}}\right) P_{-}\left(\mathbf{r}_{1}, \mathbf{r}_{2} ; N, N^{\prime}\right),
\end{aligned}
$$

in analogy with Ref. [8]. Here $z=e^{-\delta}, P\left(\mathbf{r}_{1}, \mathbf{r}_{2} ; N\right)$ is the probability of an $N$-link hull passing through $\mathbf{r}_{1}, \mathbf{r}_{2}$, and $P_{-}\left(\mathbf{r}_{1}, \mathbf{r}_{2} ; N, N^{\prime}\right)$ is the probability of an $N$-link hull passing through $\mathbf{r}_{1}$ and a different $N^{\prime}$-link hull passing through $\mathbf{r}_{2}$. In view of the cancellation of leading order terms at $z \rightarrow$ 1 , we need to consider the next, subleading term. Using again the surface critical exponent $x_{1}^{\mathrm{s}}=1 / 3$, we find, when both $\mathbf{r}_{1}$ and $\mathbf{r}_{2}$ lie at the boundary, 


$$
P\left(\mathbf{r}_{1}, \mathbf{r}_{2} ; N\right) \sim N^{-25 / 21} r^{-1 / 3}, \quad r \equiv\left|\mathbf{r}_{1}-\mathbf{r}_{2}\right| \lesssim N^{4 / 7} .
$$

Substituted in Eq. (8), this gives the value

$$
\Delta_{2}^{s}=-x_{1}^{\mathrm{s}}=-1 / 3 \text {. }
$$

A similar calculation can be done for the three-point function, with the result

$$
\Delta_{3}^{\mathrm{s}}=-3 x_{1}^{\mathrm{s}}=-1 .
$$

In this case there is no cancellation in the limit $z \rightarrow 1$, and the result conforms with the usual scaling of a three-point function at the boundary.

We have verified these predictions by direct simulation of the SQH network. To this end we have numerically diagonalized [25] the $4 L^{2} \times 4 L^{2}$ discrete time-evolution operator of the network model and selected, for each realization of disorder, two eigenstates $\psi_{i \alpha}(r)$ with lowest quasienergies $\epsilon_{i}$. We found $\Delta_{q}^{s}$ from the scaling of the moments $\left\langle\left|\psi_{i \alpha}^{2 q}(r)\right|\right\rangle$ averaged over the ensemble of $10^{4}-10^{5}$ systems. As shown in Fig. 1, the numerical data fully confirm the values of the exponents $\mu, \Delta_{2}^{\mathrm{s}}$, and $\Delta_{3}^{\mathrm{s}}$ obtained above [Eqs. (5), (9), and (10)]. Clearly, numerical simulations allow us to study also the scaling away from the analytically accessible values $q=2$, 3. In the lower panel, we show the results for the exponents $\Delta_{q}^{\mathrm{s}}$ [divided by $q(1-q)]$ in the range of $q$ between 0 and 3.5 and demonstrate their independence from the bulk exponents $\Delta_{q}^{\mathrm{b}}$.

We also show in Fig. 2 the singularity spectrum $f(\alpha)$, obtained from $\tau(q)$ by Legendre transformation. Its meaning is as follows: the number of points $r$ in the sample where the wave function intensity is $\left|\psi^{2}(r)\right| \sim L^{-\alpha}$ scales as $L^{f(\alpha)}$. The difference between the maximal values (2 vs 1) of $f^{\mathrm{b}}(\alpha)$ and $f^{\mathrm{s}}(\alpha)$ simply reflects the different dimensionalities of the bulk and the surface. On the other hand, the difference in the position of the maximum $\left(\alpha_{0}^{\mathrm{b}} \simeq 2.137\right.$ vs $\alpha_{0}^{\mathrm{s}} \simeq 2.326$ ) and in the width of the curve demonstrates that at the boundary the typical value of the intensity is suppressed, while fluctuations are stronger than in the bulk.

We have thus shown that surface MF differs significantly from that in the bulk. One can now ask the following question. Imagine that one performs a multifractal analysis for the whole sample, without separating it into "bulk" and "surface." Then would the surface exponents play any role? A naive answer is no: since the weight of surface points is down by a factor $1 / L$, one could expect that only the bulk exponents would matter. This is not true, however. To illustrate this point, we turn to an example where the whole multifractal spectrum can be studied analytically. Specifically, we will consider a 2D weakly localized metallic system (dimensionless conductance $g \gg 1$ ), which shows weak MF [5] on length scales below the localization length $\xi \sim e^{(\pi g)^{\beta}}$, where $\beta=1$ (2) for systems with preserved (respectively, broken) time-reversal symmetry. With minor modifications, the formulas below describe also the Anderson transition in $2+\epsilon$ dimensions.

The bulk multifractal spectrum of this system was obtained via the perturbative renormalization group treatment

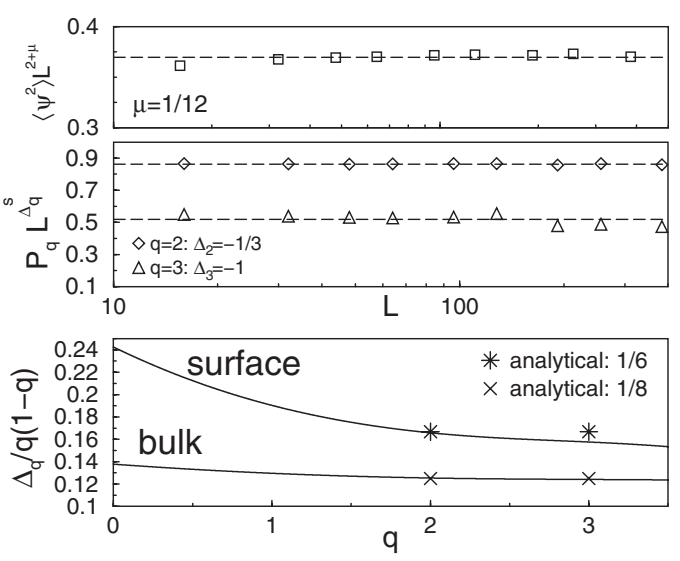

FIG. 1. Surface MF at the SQH transition. The numerical data confirm analytically found exponents (dashed lines), with deviations as low as $4 \%(\mu), 0.5 \%\left(\Delta_{2}^{\mathrm{s}}\right)$, and $6 \%\left(\Delta_{3}^{\mathrm{s}}\right)$. Lower panel: numerical results for the surface MF spectrum $\Delta_{q}^{s}$ for $0<q<$ 3.5. For comparison, the bulk spectrum $\Delta_{q}^{\mathrm{b}}$ is also shown, as well as the analytical results for $q=2,3$.

of the underlying field theory ( $\sigma$ model) $[1]$ and also within the instanton approach [5]. The result reads

$$
\tau_{q}^{\mathrm{b}}=2(q-1)+\gamma q(1-q) ; \quad \gamma=(\beta \pi g)^{-1} \ll 1 .
$$

Generalizing this calculation to the surface case, we find

$$
\tau_{q}^{\mathrm{s}}=2(q-1)+1+2 \gamma q(1-q) .
$$

The factor of 2 in front of the last (anomalous) term can be traced back to the corresponding enhancement of the return probability near the surface. Performing the Legendre transformation, we find the $f(\alpha)$ spectra,

$$
\begin{gathered}
f^{\mathrm{b}}(\alpha)=2-(\alpha-2-\gamma)^{2} / 4 \gamma, \\
f^{\mathrm{s}}(\alpha)=1-(\alpha-2-2 \gamma)^{2} / 8 \gamma .
\end{gathered}
$$

These results are illustrated in Fig. 3. When the MF in the whole sample is analyzed, the lowest of the $\tau_{q}$ exponents "wins." It is easy to see that the surface effects become dominant outside the range $q_{-}<q<q_{+}$, where $q_{ \pm} \simeq$ $\pm \gamma^{-1 / 2}$ are the roots of the equation $\tau_{q}^{\mathrm{b}}=\tau_{q}^{\mathrm{s}}$. The lower panel of Fig. 3 shows how this is translated into the $f(\alpha)$ representation. The total singularity spectrum is given by the bulk function $f^{\mathrm{b}}(\alpha)$ only for $\alpha_{+}^{\mathrm{b}}<\alpha<\alpha_{-}^{\mathrm{b}}$, where $\alpha_{ \pm}^{\mathrm{b}}-2 \simeq \mp 2 \gamma^{1 / 2}$. Outside this range the surface effects are important. Specifically, $f(\alpha)$ is equal to the surface

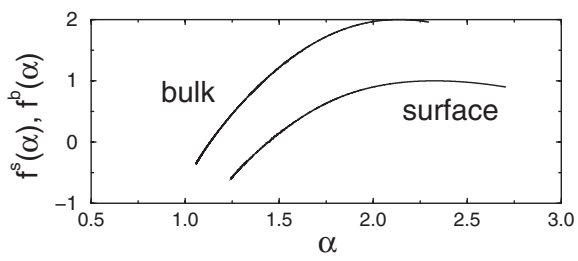

FIG. 2. Surface and bulk $f(\alpha)$ spectra at the SQH transition obtained from the data of Fig. 1. 

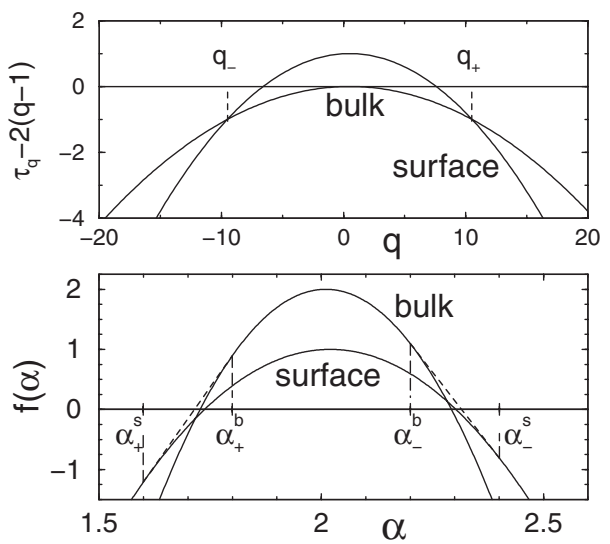

FIG. 3. Surface and bulk multifractal spectra $\tau_{q}$ and $f(\alpha)$ for a 2D metal with $\gamma=0.01$. See text for details.

spectrum $f^{\mathrm{s}}(\alpha)$ for $\alpha<\alpha_{+}^{\mathrm{s}}$ and $\alpha>\alpha_{-}^{\mathrm{s}}$, where $\alpha_{ \pm}^{\mathrm{s}}-2 \simeq$ $\mp 4 \gamma^{1 / 2}$, while in the intermediate intervals $\alpha_{+}^{\mathrm{s}}<\alpha<\alpha_{+}^{\mathrm{b}}$ and $\alpha_{-}^{\mathrm{b}}<\alpha<\alpha_{-}^{\mathrm{s}}$ its dependence on $\alpha$ becomes linear (shown by dashed lines). The latter behavior is governed by intermediate (between bulk and surface) points with a distance from the surface $r \sim L^{\beta}, 0<\beta<1$; their $f(\alpha)$ spectrum is easily found to be $f_{\beta}(\alpha)=\beta f^{\mathrm{b}}(\alpha)+(1-$ $\beta) f^{\mathrm{s}}(\alpha)$. Note that in this case the surface effects modify $f(\alpha)$ in the whole range below $f(\alpha) \simeq 1$. Therefore, the surface exponents affect the multifractal spectrum of the sample not only for rare realizations of disorder [governing the negative part of $f(\alpha)$ ] but also in a typical sample.

To summarize, we have developed the concept of surface MF for localization transitions in disordered electronic systems and have extended the notion of surface criticality to those transitions. We have calculated surface critical and multifractal exponents for the SQH transition. Considering the example of a 2D weakly localized system, we have shown that the surface criticality may crucially affect multifractal spectra in systems with boundaries. Our work opens a new direction of research in the field of Anderson and quantum Hall transitions. More generally, it is interesting to study boundary effects for MF in other stochastic systems.

We thank N. Read for initial discussions on boundary $\mathrm{MF}$ at the SQH transition. This work was supported by NSF DMR-0448820, NSF MRSEC DMR-0213745, the Alfred P. Sloan Foundation and the Research Corporation (I. A. G.), NSF DMR-00-75064 (A. W. W. L.), U.S. DOE OS No. W-31-109-ENG-38 (A. D. M,), SPP "QuantenHall-Systeme," and CFN of the DFG (F.E., A.M., A. D. M.).

*Also at Petersburg Nuclear Physics Institute, 188350 St. Petersburg, Russia.

[1] F. Wegner, Z. Phys. B 36, 209 (1980); B. L. Altshuler, V. E. Kravtsov, and I. V. Lerner, Sov. Phys. JETP 64, 1352 (1986).
[2] C. Castellani and L. Peliti, J. Phys. A 19, L429 (1986).

[3] For a review, see M. Janssen, Phys. Rep. 295, 1 (1998); A. D. Mirlin, Phys. Rep. 326, 259 (2000).

[4] M. Schreiber and H. Grussbach, Phys. Rev. Lett. 67, 607 (1991); A. Mildenberger, F. Evers, and A. D. Mirlin, Phys. Rev. B 66, 033109 (2002).

[5] V.I. Fal'ko and K. B. Efetov, Europhys. Lett. 32, 627 (1995); Phys. Rev. B 52, 17413 (1995).

[6] A. W. W. Ludwig et al., Phys. Rev. B 50, 7526 (1994); C. Mudry, C. Chamon, and X.-G. Wen, Nucl. Phys. B466, 383 (1996); H. E. Castillo et al., Phys. Rev. B 56, 10668 (1997); J.-S. Caux, N. Taniguchi, and A. M. Tsvelik, Nucl. Phys. B525, 671 (1998); Phys. Rev. Lett. 80, 1276 (1998); J.-S. Caux, Phys. Rev. Lett. 81, 4196 (1998).

[7] W. Pook and M. Janssen, Z. Phys. B 82, 295 (1991); B. Huckestein, B. Kramer, and L. Schweitzer, Surf. Sci. 263, 125 (1992); B. Huckestein, Rev. Mod. Phys. 67, 357 (1995); R. Klesse and M. Metzler, Europhys. Lett. 32, 229 (1995); Int. J. Mod. Phys. C 10, 577 (1999); M. Janssen, M. Metzler, and M. R. Zirnbauer, Phys. Rev. B 59, 15836 (1999); F. Evers, A. Mildenberger, and A. D. Mirlin, Phys. Rev. B 64, 241303 (2001).

[8] A. D. Mirlin, F. Evers, and A. Mildenberger, J. Phys. A 36, 3255 (2003).

[9] A.D. Mirlin and F. Evers, Phys. Rev. B 62, 7920 (2000).

[10] For example, K. Binder, in Phase Transitions and Critical Phenomena, edited by C. Domb and J.L. Lebowitz (Academic Press, New York 1983), Vol. 8.

[11] J. L. Cardy, Nucl. Phys. B240, 514 (1984).

[12] M. R. Zirnbauer, hep-th/9905054.

[13] M. J. Bhaseen et al., Nucl. Phys. B580, 688 (2000).

[14] B. Duplantier and A. W. W. Ludwig, Phys. Rev. Lett. 66, 247 (1991).

[15] M. R. Zirnbauer, J. Math. Phys. (N.Y.) 37, 4986 (1996); A. Altland and M. R. Zirnbauer, Phys. Rev. B 55, 1142 (1997).

[16] In analogy with the bulk, one can consider $L^{1+\mu}\left|\psi^{2}(\mathbf{r})\right|$ as a measure on the boundary and define the corresponding fractal dimensions $D_{q}^{\mathrm{s}}$ related to $\Delta_{q}^{\mathrm{s}}$ from Eq. (3) as $\Delta_{q}^{\mathrm{s}}=$ $(q-1)\left(D_{q}^{\mathrm{s}}-d+1\right)$.

[17] A. R. Subramaniam et al. (to be published).

[18] T. Senthil et al., Phys. Rev. Lett. 81, 4704 (1998); T. Senthil, J. B. Marston, and M.P. A. Fisher, Phys. Rev. B 60, 4245 (1999).

[19] I. A. Gruzberg, A. W. W. Ludwig, and N. Read, Phys. Rev. Lett. 82, 4524 (1999).

[20] E. J. Beamond, J. Cardy, and J. T. Chalker, Phys. Rev. B 65, 214301 (2002).

[21] V. Kagalovsky et al., Phys. Rev. Lett. 82, 3516 (1999).

[22] J. T. Chalker and P.D. Coddington, J. Phys. C 21, 2665 (1988).

[23] H. Saleur and M. Bauer, Nucl. Phys. B320, 591 (1989); J. Cardy, J. Phys. A 31, L105 (1998).

[24] The same scaling holds for a two-terminal conductance with a number of channels in each lead staying finite in the limit $L,\left|\mathbf{r}_{1}-\mathbf{r}_{2}\right| \rightarrow \infty$.

[25] P. R. Amestoy et al., Comput. Methods Appl. Mech. Eng. 184, 501 (2000); SIAM J. Matrix Anal. Appl. 23, 15 (2001); R. B. Lehoucq, D. Sorensen, and C. Yang, ARPACK Users Guide (SIAM, Philadelphia, 1998). 